\title{
Aerodynamics of the Mars Microprobe Entry Vehicles
}

\author{
R. A. Mitcheltree , J. N. Mossł F. M. Cheatwoodł F. A. Greeneई R. D. Braunף \\ NASA Langley Research Center, Hampton, Virginia
}

\begin{abstract}
The selection of the unique aeroshell shape for the Mars Microprobes is discussed. A description of its aerodynamics in hypersonic rarefied, hypersonic continuum, supersonic and transonic flow regimes is then presented. This description is based on Direct Simulation Monte Carlo analyses in the rarefled-flow regime, thermochemical nonequilibrium Computational Fluid Dynamics in the hypersonic regime, existing wind tunnel data in the supersonic and transonic regime, additional computational work in the transonic regime, and finally, ballistic range data. The aeroshell is shown to possess the correct combination of aerodynamic stability and drag to convert the probe's initial tumbling attitude and high velocity at atmospheric-interface into the desired surface-impact orientation and velocity.
\end{abstract}

\section{Introduction}

W HEN the Mars Surveyor mission is launched in February of 1999, it will transport not only its own lander and orbiter to Mars, but two small soil penetrators. These two Mars Microprobes ${ }^{1}$ are the second of the Deep Space missions from the New Millennium Program Office. Upon arriving at Mars, the penetrators will be released from the Surveyor spacecraft and begin a free fall to the surface. This paper focuses on the aerodynamic challenges associated with that Mars entry. In particular, the penetrators must be packaged within passive aeroshells which will carry their instrumented payloads safely through hypersonic rarefied, hypersonic continuum, supersonic, and transonic flows to an impact with the surface at prescribed velocity and attitude.

The objective of this paper is to review the decisions made in the selection of the aeroshells to fulfill these requirements, and then describe in depth the expected aerodynamics in each of the flight regimes. This description is based on Direct Simulation Monte Carlo analyses (DSMC) in the rarefied-flow regime, thermochemical nonequilibrium Computational Fluid Dy-

\footnotetext{
*Aerospace Engineer, Aerothermodynamics Branch, Aeroand Gas Dynamics Division, NASA Langley Research Center, Senior Member AIAA.

†Aerospace Engineer, Aerothermodynamics Branch, Aeroand Gas Dynamics Division, NASA Langley Research Center, Fellow AIAA.

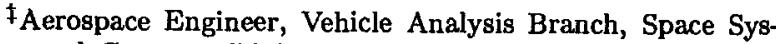
terns and Concepts Division, NASA Langley Research Center, Hampton, Va., Member AIAA.

$\$$ Aerospace Engineer, Aerothermodynamics Branch, Aeroand Gas Dynamics Division, NASA Langley Research Center, Senior Member AIAA.

Aerospace Engineer, Vehicle Analysis Branch, Space Systems and Concepts Division, NASA Langley Research Center, Senior Member AIAA.

Copyright (C)1997 by the American Institute of Aeronautics and Astronautics, Inc. No copyright is asserted in the United States under Title 17, U.S. Code. The U.S. Government has a royalty-free license to exercise all rights under the copyright claimed herein for governmental purposes. All other rights are reserved by the copyright owner.
}

namics (CFD) in the hypersonic regime, existing wind tunnel data in the supersonic and transonic regime, additional CFD in the transonic regime, and finally, ballistic range data.

\section{Selection of the Aeroshell}

Selection of the aeroshell for Mars Microprobe requires consideration of the unique objectives of the mission. An enclosure is required which will safely deliver the penetrator payload through entry to impact with the surface. The aeroshell must decelerate the vehicle during its descent to a prescribed impact velocity. It must possess sufficient stability to correct an initial tumbling attitude to one of forward-facing flight early in the trajectory, and it must maintain that orientation within small tolerances until impact. This attitude control must be accomplished passivly since there is no accomodation for active controls. Finally, the aeroshell must protect the payload from intense aerodynamic heating. The basis for this selection is discussed in the following paragraphs.

The Viking ${ }^{2}$ and Mars Pathfinder ${ }^{3}$ landers, as well as the Pioneer-Venus ${ }^{4}$ and Galileo ${ }^{5}$ probes used spherically blunted cones for their forebody. Selection of the appropriate cone angle involves trade-offs between drag, heating, stability, and overall size. Blunter (i.e., larger half-angle) cones exhibit more drag for a given surface area as well as lower heating; sharper cones possess more stability. Both Pioneer-Venus and Galileo used 45-degree half-angle cones, but these missions were entering atmospheres much denser than Mars. The two Mars missions (Viking and Pathfinder) require the higher-drag properties of 70-degree spherecones to decelerate their heavy landers at sufficiently high altitudes to permit parachute deployment. Mars Microprobe is light (less than $3 \mathrm{~kg}$ ) and must impact the surface with sufficient speed for penetrator insertion. In addition, Microprobe requires the highest possible stability.

The impact-velocity problem can be discussed in 
terms of the ballistic coefficient $(B)$.

$$
B=\frac{M}{C_{D} A}
$$

where $M$ is the vehicle mass, $A$ is the frontal area, and $C_{D}$ is the drag coefficient. Figure 1 compares the expected impact velocity versus ballistic coefficient for this Mars entry from three degree-of-freedom entry simulations. The range of acceptable impact speeds is shown in the figure. For a velocity of $160 \mathrm{~m} / \mathrm{s}$, a ballistic coefficient near $28 \mathrm{~kg} / \mathrm{m}^{2}$ is necessary. The current mass estimate for Microprobe is $2.4 \mathrm{~kg}$, and the size which can be attached to the Mars Surveyor spacecraft has diameter (D) of $0.325 \mathrm{~m}(\mathrm{~A}=0.08296$ $m^{2}$ ), thus the drag coefficient must be around 1.0: the value for a 45-degree cone. [Since this work was performed the diameter had increased to $0.35 \mathrm{~m}$ and the allowable mass has increased to $2.8 \mathrm{~kg}$ which still suggests a drag coefficient near 1.0.]

The extent of spherical blunting of the nose has a minimal effect on the drag coefficient for a 45-degree half-angle cone. However, Fig. 2 reveals that increased nose bluntness $\left(R_{n} / D\right.$, where $R_{n}$ is the nose radius) decreases the maximum stagnation point heating rate $(q)$, since heat rate varies as the inverse square of the effective nose radius. Unfortunately, increased nose bluntness also decreases static stability, as shown by the decreasing negative values for the slope of the moment curve $\left(C_{m, \alpha}\right.$ from Mach 1.65 wind tunnel measurements in Ref. 6). Selecting the appropriate extent of nose bluntness is a trade-off between heating and stability. For Microprobe, a nose radius equal to half of the overall vehicle's base radius $\left(R_{n} / D=0.25\right)$ is chosen. This is the same ratio as used in the PioneerVenus and Galileo probes. In an analogous manner, rounding the vehicle's shoulders decreases the heating at that location. However, rounding the shoulders decreases drag and stability. The compromise is to again use the Pioneer-Venus values: shoulder radius $\left(R_{s}\right)$ equal to one-tenth the nose radius. It is possible to optimize the nose and shoulder radii for the Microprobe mission, but these previously used ratios appear adequate, and their selection allows the use of an extensive body of existing aerodynamic data. The geometry of the Pioneer-Venus small probes is shown in Fig. 3.

The hemispherical afterbody serves two purposes. First, since the vehicle's initial attitude is tumbling, it may encounter the atmosphere while traveling backwards. A hemispherical afterbody with its center at the vehicle's center-of-gravity is not stable at this backward orientation and will foster rotation to a forwardfacing attitude. Second, the shape has been shown to decrease the dynamic instability observed in blunt vehicles traversing the transonic flight regime ${ }^{8}$. Selection of the large hemispherical afterbody for Microprobe does not rely on previous mission's designs, but is sim- ilar to the one used in the PAET experiment ${ }^{7}$.

Regarding the backward stability issue, PioneerVenus, Galileo, Viking, and Mars Pathfinder were all hypersonically stable in either a forward or backward orientation. To avoid a backward entry, each were oriented nose first and spin-stabilized to assure a nose-first attitude at atmospheric interface. Since spin-stabilization is not an option for Microprobe, an afterbody which assures the vehicle does not trim in a rearward facing attitude must be chosen.

The desired impact velocity of Mars Microprobe is just below Mach 1. Unfortunately, aeroshells like the 45-degree and 70-degree blunted cones mentioned above suffer a dynamic instability at small angles of attack in the Mach 1.0 to 2.0 range. ${ }^{2,8,9,10}$. That is, though they remain statically stable as they traverse that Mach range, an increase in incidence angles is observed. Since the instability is restricted to anglesof-attack less than 5 degrees, the increase in incidence is bounded and results in a wobbling motion. Unfortunately for Microprobe, surface impact near Mach 1.0 means this instability interferes with its requirement of small angle-of-attack at impact. Both Viking and Mars Pathfinder deployed (or plan to deploy) parachutes at supersonic speeds to avoid this dynamics problem. Galileo unexpectedly traversed the transonic flight regime and flight data ${ }^{10}$ indicate a growth in incidence angles beginning at Mach 2.0 and increasing to a maximum value near 15 degrees at Mach 1.0.

The source of the dynamic instability is still debated. Sammonds ${ }^{8}$ argued that a hemispherical afterbody centered about the center-of-gravity would eliminate this problem since afterbody pressure forces would be directed through the c.g. such that asymmetric pressure distributions there would produce no pitching motions. He demonstrated this fact at Mach numbers up to 1.2. While it is unproven for larger Mach numbers, it is arguable that a hemispherical afterbody will decrease the dynamic instability.

Based on the above discussion, the forebody geometry of the Mars Microprobe is specified to be a 45-degree sphere-cone with $R_{n}=0.08125 \mathrm{~m}, R_{s}=$ $0.008125 \mathrm{~m}$, and $\mathrm{D}=0.325 \mathrm{~m}$. The afterbody shape is a hemispherical section with radius $0.174 \mathrm{~m}$ which is centered about the expected c.g. location of the vehicle. The geometry is shown in Fig. 4.

\section{Trajectory}

Detailed analysis of the aerodynamics requires knowledge of the expected trajectory. A preliminary aerodynamic description of the Mars Microprobe was constructed using free-molecular aerodynamics, Newtonian aerodynamics, and Pioneer-Venus supersonic and transonic wind tunnel data. This description was then used in a preliminary six degree-of-freedom (6DOF) trajectory simulation to create an estimated nominal trajectory. Altitude and Mach number ver- 
sus velocity from that trajectory are presented in Fig. 5. Points from this trajectory in each flight regime were then selected for more detailed analysis.

\section{Free-Molecular Flow}

Free-molecular flow assumes there are no collisions between gas molecules in the flow field. The surface is impacted by free-stream particles which are diffusely reflected after full thermal accommodation. Unlike hypersonic-continuum aerodynamics where forces exerted on the body are primarily the integrated effect of surface pressures, free-molecular flow aerodynamics contain a significant contribution from shear stress.

The two Mars Microprobes are to be released from the host spacecraft three minutes prior to atmospheric interface. As the tumbling spacecraft approach the outer reaches of the atmosphere, they will encounter widely spaced molecules. Surface impacts of these molecules will exert the first aerodynamic forces on the entry vehicle. Knudsen number $(K n)$ is the ratio of the quiescent gas's mean free path to the vehicle's diameter. At the outer reaches of the atmosphere, $K n$ is large. As long as it remains of order 10 or larger, the associated aerodynamic forces can be computed by free-molecular-flow methods.

The free-molecular flow aerodynamics for the Mars Microprobe entry vehicles are presented in the form of nondimensional coefficients in Fig. 6. Since the geometry is axisymmetric, the static aerodynamics can be described by an axial-force coefficient $\left(C_{A}\right)$, a normalforce coefficient $\left(C_{N}\right)$, and a moment coefficient $\left(C_{m}\right)$. The reference area is the frontal area of the aeroshell $\left(0.08296 \mathrm{~m}^{2}\right)$, and the reference length is the diameter $(0.325 \mathrm{~m})$. Unless otherwise specified, moments are taken about the actual nose of the vehicle. For a c.g. location $0.24 \mathrm{D}$ back from the nose or less, the vehicle is statically stable only when facing forward.

\section{Hypersonic Transitional and Continuum Aerodynamics}

As the descent continues into the upper atmosphere, the mean free path (and $K n$ ) decrease and collisions between particles must be accounted for in describing forces on the vehicles. This is the transitional flow regime. Even lower (below $55 \mathrm{~km}$ altitude for Microprobe), $K n$ drops below 0.001 and continuum methods can be used to describe the flow about, and the forces on, the vehicle. Figure 7 presents $K n$ versus altitude for the high altitude portion of the Microprobe trajectory.

For a given $K n$, the transitional aerodynamics can be computed using DSMC methods ${ }^{11}$. Axisymmetric solutions at eight points during the high-altitude portion of the trajectory were computed. The predictions for $C_{A}$ are listed in Table 1. and seven of the points are plotted in Fig. 8. The figure also contains the free-
Table 1 Axisymmetric Results from DSMC.

\begin{tabular}{|c|c|c|c|c|c|}
\hline$t(s)$ & Alt $(\mathrm{km})$ & $\bar{M}$ & $\mathrm{~V}(\mathrm{~m} / \mathrm{s})$ & $\overline{K n}$ & $C_{A}$ \\
\hline 10.4 & 126.7 & 33.3 & 6909. & 86.5 & 2.054 \\
\hline 20.3 & 113.2 & 35.1 & 6916. & 10.08 & 2.010 \\
\hline 31.8 & 100.6 & 37.4 & 6921. & 1.25 & 1.901 \\
\hline 38.0 & 91.4 & 39.1 & 6923. & 0.305 & 1.771 \\
\hline 48.0 & 80.3 & 37.7 & 6908. & $5.46 \mathrm{e}-02$ & 1.536 \\
\hline 50.5 & 73.4 & 35.9 & 6884. & $2.45 \mathrm{e}-02$ & 1.352 \\
\hline 65.0 & 64.3 & 35.6 & 6751. & $6.78 \mathrm{e}-03$ & 1.190 \\
\hline 77.2 & 54.9 & 31.8 & 6168. & $1.83 \mathrm{e}-03$ & 1.108 \\
\hline
\end{tabular}

molecular prediction as well as eight continuum CFD solutions discussed later.

Values from a bridging function, selected as appropriate to recreate the data when $10.0>K n>0.001$, are included in Fig. 8. The bridging function for the axial-coefficient is:

$$
\begin{gathered}
C_{A}=C_{A, c}+\left(C_{A, f m}-C_{A, c}\right) \sin ^{2} \phi \\
\phi=\pi\left(\frac{3}{8}+\frac{1}{8} \log _{10} K n\right)
\end{gathered}
$$

$C_{A, f m}$ and $C_{A, c}$ are the axial coefficients at the freemolecular and continuum limits, respectively. This equation approximates the monotonic decrease in $C_{A}$ across the transitional regime. There is a region around $K n=0.01$ where both DSMC and continuum CFD methods can predict the axial coefficient. Above $K n$ of 0.01 , the continuum CFD calculations become increasingly suspect. (Slip boundary conditions would be required in continuum methods to predict the flowfields at such low density conditions.)

Three-dimensional DSMC solutions over a range of angles-of-attack were computed at three of the points from Table 1. The results (for $C_{A}, C_{N}$, and $C_{m}$ ) of these solutions are listed in Table 2 and compared with bridging functions similar to Eqns. 2-3 in Figs. 9-11. The bridging functions approximate the predictions of $C_{A}, C_{N}$, and $C_{m}$ at angle-of-attack in this regime.

In the hypersonic continuum regime, the Langley Aerothermodynamic Upwind Relaxation Algorithm (LAURA) CFD tool can describe the static aerodynamics of Microprobe. LAURA is an upwind-biased, point-implicit relaxation algorithm ${ }^{12}$ for obtaining the numerical solution to the Navier-Stokes equations for three-dimensional viscous hypersonic flows in thermochemical nonequilibrium. The Mars atmosphere version of the code ${ }^{13}$ contains an 8-species $\mathrm{CO}_{2}-\mathrm{N}_{2}$ chemical-kinetics model. This is the same computational tool used to describe the aerodynamics of Mars Pathfinder ${ }^{14,15}$. Predictions from the code have been shown to be in agreement with Viking flight data ${ }^{15}$.

LAURA solutions are computed at eight points in the trajectory's hypersonic continuum regime. Table 
Table 2 Three Dimensional Results from DSMC.

\begin{tabular}{|r|c|c|c|c|}
\hline Alt(km) & $\alpha(\mathrm{deg})$ & $C_{A}$ & $C_{N}$ & $C_{m}$ \\
\hline 126.7 & 0 & 2.054 & 0.000 & .0001 \\
126.7 & 5 & 2.040 & 0.176 & -.0718 \\
126.7 & 15 & 1.921 & 0.505 & -.2061 \\
126.7 & 30 & 1.574 & 0.891 & -.3700 \\
126.7 & 45 & 1.118 & 1.097 & -.4755 \\
126.7 & 60 & 0.723 & 1.225 & -.5593 \\
126.7 & 75 & 0.360 & 1.306 & -.5413 \\
126.7 & 90 & .0015 & 1.336 & -.5469 \\
126.7 & 105 & -0.358 & 1.305 & -.5370 \\
126.7 & 120 & -0.725 & 1.224 & -.5152 \\
126.7 & 135 & -1.122 & 1.095 & -.4672 \\
126.7 & 150 & -1.570 & 0.890 & -.3626 \\
126.7 & 165 & -1.921 & 0.506 & -.2040 \\
126.7 & 180 & -2.054 & 0.000 & -.0002 \\
\hline 100.6 & 0 & 1.901 & 0.000 & -.0004 \\
100.6 & 5 & 1.891 & 0.156 & -.0657 \\
100.6 & 15 & 1.788 & 0.452 & -.1910 \\
100.6 & 30 & 1.470 & 0.794 & -.3403 \\
100.6 & 45 & 1.071 & 0.991 & -.4391 \\
100.6 & 60 & 0.716 & 1.126 & -.4911 \\
100.6 & 75 & 0.369 & 1.222 & -.5161 \\
100.6 & 90 & 0.008 & 1.252 & -.5138 \\
\hline 80.3 & 0 & 1.536 & 0.001 & -.0013 \\
80.3 & 5 & 1.520 & 0.128 & -.0580 \\
80.3 & 15 & 1.424 & 0.360 & -.1650 \\
80.3 & 30 & 1.180 & 0.607 & -.2838 \\
80.3 & 45 & 0.904 & 0.734 & -.3525 \\
80.3 & 60 & 0.656 & 0.846 & -.4035 \\
\hline & & & & \\
\hline
\end{tabular}

Table 3 Axisymmetric Results from LAURA CFD.

\begin{tabular}{||l||r|c|c||c||l||}
\hline $\mathrm{t}(\mathrm{s})$ & Alt $(\mathrm{km})$ & $\mathrm{M}$ & $\mathrm{V}(\mathrm{m} / \mathrm{s})$ & $K n$ & $C_{A}$ \\
\hline 48 & 80.5 & 37.7 & 6913 & $6.1 \mathrm{e}-02$ & 1.598 \\
55 & 73.4 & 35.9 & 6884 & $2.5 \mathrm{e}-02$ & 1.378 \\
72 & 55.8 & 32.8 & 6271 & $2.0 \mathrm{e}-03$ & 1.086 \\
82 & 48.7 & 25.9 & 5419 & $1.0 \mathrm{e}-03$ & 1.061 \\
92 & 43.3 & 19.9 & 4327 & $6.2 \mathrm{e}-04$ & 1.049 \\
116 & 34.9 & 10.0 & 2206 & $2.6 \mathrm{e}-04$ & 1.027 \\
125 & 32.6 & 7.5 & 1665 & $2.1 \mathrm{e}-04$ & 1.028 \\
142 & 29.5 & 5.0 & 1121 & $1.6 \mathrm{e}-04$ & 1.035 \\
\hline
\end{tabular}

3 presents the zero-degree angle-of-attack $C_{A}$ results. Additional forebody solutions are computed at $\mathbf{1 0} \mathrm{de}-$ grees angle-of-attack for six of these trajectory points. Those results are listed in Table 4.

The variation in axial coefficient at zero degrees angle-of-attack across the hypersonic-continuum regime is presented in Fig. 12. Little change in $C_{A}$ occurs across the Mach range. The variation at 10 degrees angle-of-attack of the normal-force and mo-
Table 4 3-D Results from LAURA, $\alpha=10^{\circ}$.

\begin{tabular}{|c||c||c||c||r||}
\hline $\mathrm{t}(\mathrm{s})$ & $\mathrm{M}$ & $C_{A}$ & $C_{N}$ & $C_{m}$ \\
\hline 72 & 32.8 & 1.0281 & 0.1687 & -0.1106 \\
82 & 25.9 & 1.0353 & 0.1689 & -0.1103 \\
92 & 19.9 & 1.0141 & 0.1706 & -0.1125 \\
116 & 10.0 & 1.0016 & 0.1691 & -0.1115 \\
126 & 7.5 & 0.9987 & 0.1668 & -0.1125 \\
142 & 5.0 & 1.0133 & 0.1635 & -0.1064 \\
\hline
\end{tabular}

ment coefficients are given in Figs. 13 and 14. Again, the aerodynamic coefficients vary little down to Mach 5. (The aerodynamics below Mach 5 are discussed in subsequent sections.) The values predicted from the Newtonian flow approximation are included in Figs. 12-14. Newtonian flow assumes that the free-stream flow is turned parallel to the surface. Local pressure is then a function only of the local surface inclination relative to the free stream. While the simple Newtonian assumption agrees with the CFD precitions for the axial and normal-force coefficients across the hypersonic regime, it under predicts the the absolute value of the moment coefficient by as much as 14 percent.

At the higher Mach numbers, the bow shock is sufficiently energetic to initiate dissociation of the triatomic $\mathrm{CO}_{2}$ molecule. The degree of $\mathrm{CO}_{2}$ dissociation on the stagnation streamline associated with four LAURA solutions is shown in Fig. 15. At Mach 33, $\mathrm{CO}_{2}$ dissociates from its free-stream mass fraction of 0.97 to 0.19 at the edge of the boundary layer. (The fully catalytic wall boundary condition forces complete recombination of the dissociated molecules.) Lesser degrees of dissociation occur with decreasing Mach number. No dissociation is predicted at Mach 10 . When dissociation occurs, the residence time of the gas across the thin shock layer is insufficient to allow the gas to equilibrate. This phenomena is a result of the small length scales associated with Microprobe. There does not appear to be a Mach range where an equilibrium gas assumption would be useful in predicting the flowfield about Microprobe.

Figure 15 also shows the associated shock standoff distances. The distance at Mach 33 is large due to rarefaction effects, the standoff distance reaches a minimum below Mach 26, then grows with decreasing speeds.

\section{Supersonic Aerodynamics}

In the supersonic regime, existing wind tunnel data can be used to describe the aerodynamics. Nichols and Nirengarten ${ }^{6}$ tested 45-degree half-angle cones in the Jet Propulsion Laboratory (JPL) 21-inch supersonic wind tunnel at Mach 3.0, 2.0, and 1.65 and in the JPL 20-inch hypersonic wind tunnel at Mach 5.0. The tests were performed in air at Reynolds number (based on diameter of 3.0 in. models) of $0.3 \times 10^{6}$ 
in the hypersonic tunnel and $1.0 \times 10^{6}$ in the supersonic tunnel. The flight Reynolds numbers expected for Mars Microprobe in this speed range are below 0.1 $\times 10^{6}$ in the Mars $\mathrm{CO}_{2}$ atmosphere. While the flow in this regime should be that of a perfect gas, the ratio of specific heats for air at 1.4 is higher than the Mars atmosphere value of 1.33. In addition, these measurements examined a model which did not include the hemispherical afterbody. Afterbody effects are negligible for small angles-of-attack above Mach 5 (which allowed the forebody-only CFD used in the hypersonic aerodynamics description). Below Mach 5, however, the afterbody's influence increases. As shown in Figs. 12-14, the Mach 5 measurements compare well with the LAURA CFD calculations.

Figures 16-18 focus on the Mach range below 5 and include wind tunnel, ballistic range, and CFD data. The solid line in the figures labeled "database" is included to show trends across the Mach range. The figures reveal a marked change in the aerodynamics across the supersonic and transonic regimes. The change is a result of the sonic line shifting from the nose region to the shoulder region. At Mach 5, the subsonic bubble remains on the spherical nose even at 10 degrees incidence. By Mach 2, the sonic line has shifted to the shoulder even at zero degrees incidence. At this condition, the entire forebody shock layer is subsonic flow.

Pressure distributions, and aerodynamic forces are affected by the sonic character of the shock layer. When the later above the flank is supersonic, the pressure distributions there is flat (a characteristic of conical flow). When the shock layer is subsonic, the elliptic nature of the flow results in higher, more rounded, pressure distributions. The net effect on the resulting forces is that the axial force increases while normal-force and moment coefficients decrease. This combination results in a decrease in the static stability margin of the vehicle.

If the vehicle's c.g. does not lie on the geometric symmetry line, the vehicle will trim at a non-zero angle-of-attack. As the Mach range between 5 and 2 is traversed, the decrease in static stability would introduce pitching motions for such an offset c.g. since the vehicle would then trim at a larger angle-of-attack. Since Microprobe has strict requirements on angleof-attack at impact (and impact occurs shortly after traversing this Mach range), c.g. offset should be minimized to avoid the introduction of pitching motions late in the trajectory.

\section{Transonic Aerodynamics}

Figures 16-18 also contain the transonic aerodynamics. The probe's impact speed at just below Mach 1 , and its requirement that this impact occur while at small angle-of-attack demand the best possible description of the aeroshell's aerodynamics in this speed regime. Unfortunately, transonic aerodynamics are complex, and testing and computations are difficult (as well as expensive). The description of Microprobe's transonic aerodynamics combines existing wind tunnel data, new CFD, and recent ballistic-range tests performed for Microprobe.

Wind tunnel data ${ }^{2,16}$ in the transonic regime were obtained in support of the Pioneer-Venus small probes which have the same forebody as Microprobe. Both vehicles have hemispherical afterbodies (Figs. 3 and 4), though Microprobe's is larger. In Ref. 16, static aerodynamics for Pioneer-Venus models were measured at Mach $0.2,0.5,0.8,0.95,1.1,1.2$, and 1.35 in Langley's 8-foot transonic wind tunnel, and Mach 1.5, 1.8, and 2.16 in the Langley Unitary Plan wind tunnel. The reference describes testing problems encountered at Mach numbers 1.2, 1.35, and 1.5. Figures 16-18 exclude the data from those Mach numbers.

Figure 16 also contains CFD results at five points between Mach 0.6 and Mach 2.2 using the TLNS3D (Thin Layer Navier Stokes 3-Dimensional) code ${ }^{17}$. These calculations are about the Microprobe geometry for conditions coincident with the Brooks (Ref. 16) wind tunnel data. The results presented were preceded by an extensive grid-resolution study to resolve the wake region. The CFD calculations span the range where the Brooks study encountered problems with reflected wall-shock interactions. In an effort to assess the usefulness of the Pioneer-Venus wind-tunnel measurements in the transonic regime, additional TLNS3D CFD was computed at the same five conditions for the Pioneer-Venus geometry. The axial-force coeffcients for the two geometries are compared in Fig. 19. Higher base pressures on the large Microprobe afterbody result in a decrease (as large as 6 percent) in the predicted drag coefficient for the Microprobe geometry relative to the Pioneer-Venus geometry.

Ballistic range tests of the Microprobe geometry were performed at Wright Patterson Laboratory's Aeroballistic Research Facility (ARF) at Eglin Air Force Base. The test were concentrated around Mach 0.8 and 1.4. The primary goal of these tests were to establish the dynamic stability of the vehicle (discussed in the next section). Static aerodynamics, however, were also measured and are included in Figs. 16-18. With the exception of $C_{N}$, there is good agreement among the ballistic range measurements, the CFD, and the wind tunnel data. The ballistic range is accurate at measuring axial forces, it has difficulty resolving the small normal forces associated with a blunt body such as Microprobe.

The Reynolds numbers based on diameter for this wind tunnel and ballistic range data is between 0.5 and $3.0 \times 10^{6}$. The expected flight conditions have Reynolds numbers between 50,000 to 100,000 for this speed range. (Additional transonic aerodynamics have recently been measured in the TsNIIMash U-21 wind 
tunnel at the correct Reynolds number and will appear in a subsequent publication.)

\section{Dynamic Derivatives}

Detailed measurement of the dynamic derivatives for Microprobe over its entire trajectory to a level of accuracy necessary for precise 6-DOF simulation is difficult given the time and cost restraints on the project. Microprobe must impact the surface while flying at small angle-of-attack. This impact will occur around Mach 0.8. The primary concern for dynamic stability, therfore, is in the transonic flight regime. Through use of existing data measured for the Mars Viking and Pioneer-Venus missions in conjunction with the transonic dynamic data measured in the Eglin ARF ballistic range, an assessment of the transonic dynamic stability of Microprobe can be made.

In preparation for the Mars Viking mission, Uselton et al. ${ }^{9}$ performed extensive wind tunnel measurements of the dynamic stability of 60- and 70-degree halfangle cones using a forced-oscillation technique. That reference concludes there is no appreciable difference between the dynamic response of the two cones when the c.g. location of the 70 degree cone is more forward than that of the 60 degree cone. The reference also observes a decrease in dynamic stability for a given cone angle when the c.g. is moved further from the nose. Based on these two observations, it is arguable that the 60 degree cone is a more dynamically stable shape than the 70-degree cone, but this increase in stability can be offset by an aft shift in the c.g. If this reasoning is extrapolated to 45 -degree cones, a 45 - degree cone should exhibit more dynamic stability than a 60-degree cone. Therefore, the Uselton 60-degree cone data should represent a conservative description of the dynamic performance of Microprobe. Figures 20-22 present the angle-of-attack variation of the dynamic derivatives at three Mach numbers in the transonic regime. Since the geometry is symmetric, the dynamic derivatives can be specified by a single parameter, $\left(C_{m, q}+C_{m, \dot{\alpha}}\right)$.

Dynamic tests in support of Pioneer-Venus were performed in the Langley 8 foot transonic wind tunnel using a similar forced oscillation technique ${ }^{4}$. The pioneer Venus small probe geometry (Fig. 3) has the same forebody as Microprobe. The c.g. location is $0.22 \mathrm{D}$ back from the nose for the Pioneer-Venus tests. Those tests were restricted to Mach numbers below 1.3 so representative values are included only in Figs. 20 and 21.

Figures 20 and 22 also contain the Eglin ARF ballistic range data for the two Mach numbers (0.8 and 1.4) examined. The ballistic range did not distinguish an angle-of-attack variation in the dynamic damping, those results (two separate measurements for each Mach number) are presented as the straight dotted lines in the figures.
At Mach 0.8 (Fig. 20), all three data sources predict dynamic stability (a negative quantity) across the angle-of-attack range. The figure shows that a 45-degree cone possess a higher degree of dynamic stability than the 60-degree cone. The Eglin ARF data is in good agreement with the wind tunnel data.

The instability at small angles-of-attack for Mach 1.2 and 1.4 is indicated by the positive values for $\left(C_{m, q}+C_{m, \dot{\alpha}}\right)$ in Figs. 21 and 22. The ARF Ballistic range data at Mach 1.3-1.45 did not detect a transonic dynamic instability for the Microprobe geometry. This may be due to Microprobe's hemispherical afterbody which, as reported by Sammonds, can eliminate or reduce the transonic dynamic instability.

Accurate 6-DOF simulation over the entire entry trajectory requires knowledge of the dynamics at higher speeds. The Uselton measurements are restricted to Mach numbers at 3.0 and below. For larger Mach numbers, other ballistic range tests on blunt bodies $^{18}$ do not discern significant changes with Mach number. Thus, Mach 3.0 values for a 60-degree cone could be used as representative of the higher speed performance. Past experience with 6-DOF simulations have also indicated that large variations in the dynamic derivatives at the higher Mach numbers have little effect on the hypersonic-flight dynamics of the vehicle.

As with the supersonic and transonic data, there is a large difference between the Reynolds numbers for the dynamic data discussed above and the expected flight values for Microprobe. Uselton ${ }^{9}$ examined Reynolds number effects on dynamic stability for 70-degree cones. No clear trend was revealed. (Additional dynamic windtunnel test in the TsNIIMash U-21 at the correct Reynolds number will be discussed in a subsequent publication.)

\section{Conclusions}

Selection of the aeroshell for Mars Microprobe involved consideration of the unique objectives of the mission. An enclosure was required which possessed the appropriate drag and stability characteristics to safely deliver the penetrator payload through entry to impact with the surface. A 45-degree half-angle cone with rounded nose and shoulder was shown to be a suitable candidate for the forebody shape. A hemispherical afterbody centered about the vehicle's center-of-gravity location was chosen for the afterbody.

The aerodynamics of this aeroshell are discussed in each of the expected flight regimes during its Mars entry. This description makes use of Direct Simulation Monte Carlo (DSMC) analyses, Computational Fluid Dynamics (CFD), existing wind tunnel data, and new ballistic range data.

Free-molecular flow aerodynamics indicate that the vehicle is statically unstable backwards. If the tumbling vehicle has a rearward orientation when it en- 
counters the atmosphere, it will reorient itself to a forward-facing attitude.

In the high-altitude transitional flow regime, a bridging function is shown to approximate the variation of the aerodynamic coefficients from their freemolecular values (at $K n=10.0$ ), to their hypersonic continuum values ( $K n$ of 0.001 ). In addition, good agreement between CFD and DSMC predictions for axial coefficient is observed for $2.0 \times 10^{-3}$ i $K n$ i $3.0 \times 10^{-2}$.

In the hypersonic-continuum regime, the Newtonian assumption is shown to be a fair estimate for the axial and normal force coefficients for angles-of-attack up to 10 degrees, but it under predicts the magnitude of the moment coefficient by as much as 14 percent.

In the hypersonic regime, the small length scales associated with the forebody shock layer are insufficient to allow the dissociation of $\mathrm{CO}_{2}$ to reach completion. Accurate description of the species profiles requires inclusion of chemical nonequilibrium effects for all cases in which chemical reactions occur. At Mach 10 and below, no $\mathrm{CO}_{2}$ dissociation is predicted.

Around Mach 5, the sonic line shifts from the nose to the shoulder resulting in an increase in axial coefficient and a decrease in static stability of the vehicle.

Ballistic range tests reveal that the combination of 45-degree cone, forward c.g. location, and hemispherical afterbody has reduced the dynamic instability often suffered by large-angle cones traversing the transonic-flow regime.

\section{Acknowledgments}

Thanks are extended to Mr. Eric Slimko of the Jet Propulsion Laboratory for many helpful discussions. Dr. Didier Rault of Langley Research Center provided the free-molecular flow aerodynamics presented herein.

\section{References}

${ }^{1}$ Braun, R. D., Mitcheltree, R. A., and Cheatwood, F. M., "Mars Microprobe Entry Analysis," Proceedings from the 1997 IEEE Aerospace Conference, Feb. 1997.

${ }^{2} \mathrm{Kirk}$,

B., Intrieri, P. F., and Seiff A., "Aerodynamic Behavior of the Viking Entry Vehicle: Ground Test and Flight Results," Journal of Spacecraft and Rockets, Vol 15., No. 4., July-Aug 1978., pp208-212.

${ }^{3}$ Spear, A. J., Freeman, D. C., and Braun, R. D., "Mars Pathfinder Status at Launch," IAF Paper 96-Q.3.02, Bejing, China, Oct., 1996.

${ }^{4}$ Weinberg, S. A., "Final Data Report - PioneerVenus Static and Dynamic Transonic Wind Tunnel Tests," 9154-TDM-75-013, General Electric Corp, July 11, 1975.

${ }^{5}$ Moss, J. N., LeBeau, G. J., Blanchard, R. C., and Price, J. M., "Rarefaction Effects on the Galileo Probe Aerodynamics," 20th International Symposium on Rarefied Gas Dynamics, Beijing, China, Aug., 1996.
${ }^{6}$ Nichols,

O., and Nierengarten, E. A., "Aerodynamics Characteristics of Blunted Bodies," JPL TR 32-677., 1964.

${ }^{7}$ Seiff, A., Reese, D. E., Sommer, S. C., Kirk, D. B., Whiting, E. E., and Niemann, H. B., "PAET, An Entry Probe Experiment in the Earth's Atmosphere," Icarus, Vol. 18, No. 4, April, 1973, pp. 525-563.

${ }^{8}$ Sammonds, R. I., "Dynamics of High Drag Probe Shapes at Transonic Speeds," NASA TN D-6489, Sept, 1971.

${ }^{9}$ Uselton B. L., Shadow, T. O., Mansfield, A. C., "Damping in Pitch Derivatives of 120 and 140 Deg Blunted Cones at Mach Numbers 0.6 through 3.0," AEDC TR-70-49. 1970.

${ }^{10}$ Blanchard, R., private communication, NASA Langley Research Center, April, 1996.

${ }^{11}$ Bird, G. A., "The G2/A3 Program Users Manual," G.A.B. Consulting Pty Ltd., Killara, N.S.W., Australia, March 1992.

${ }^{12}$ Gnoffo, P. A., "An Upwind-Biased, Point-Implicit Relaxation Algorithm for Viscous, Compressible Perfect-Gas Flows," NASA TP-2953, Feb. 1990.

${ }^{13}$ Mitcheltree, R. A., "Aerothermodynamic Methods for a Mars Environmental Survey Mars Entry," Journal of Spacecraft and Rockets, Vol. 31, No. 3, MayJune, 1994, pp. 516-523.

${ }^{14}$ Braun, R. D., Powell, R. W., Engelund, W. C., Gnoffo, P. A., Weilmuenster, K. J., and Mitcheltree, R. A., "Mars Pathfinder Six-Degree-of-Freedom Entry Analysis," Journal of Spacecraft and Rockets, Vol. 32, No. 6., Nov-Dec., 1996, pp. 993-1000.

${ }^{15}$ Gnoffo, P. A., Weilmuenster, K. J., Braun, R. D., and Cruz, C. I., "Influence of Sonic Line Location on Mars Pathfinder Aerodynamics," Journal of Spacecraft and Rockets, Vol. 33, No. 2, Mar-Apr. 1996, pp. 169177.

${ }^{16}$ Brooks, J. D., "Some Anomalies Observed in Wind Tunnel Tests of a Blunt Body at Transonic and Supersonic Speeds," NASA TN D-8237, June, 1976.

${ }^{17}$ Vatsa, V. N., Turkel, E., and Abolhassani, J. S., "Extension of Multigrid Methodology to Supersonic/Hypersonic 3-D Viscous Flows," NASA Contractor Report 187612, Aug., 1991.

${ }^{18}$ Krumins, M. V., "A Ballistic Range Study of the Aerodynamic Characteristics of Mars Probe/Lander Shapes," AIAA Paper 67-167, Jan 1967. 


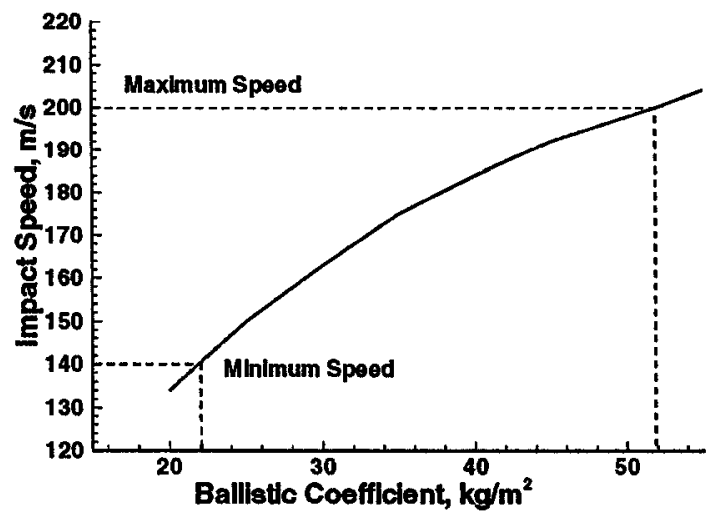

Fig. 1 Ballistic coefficient required for different impact speeds.

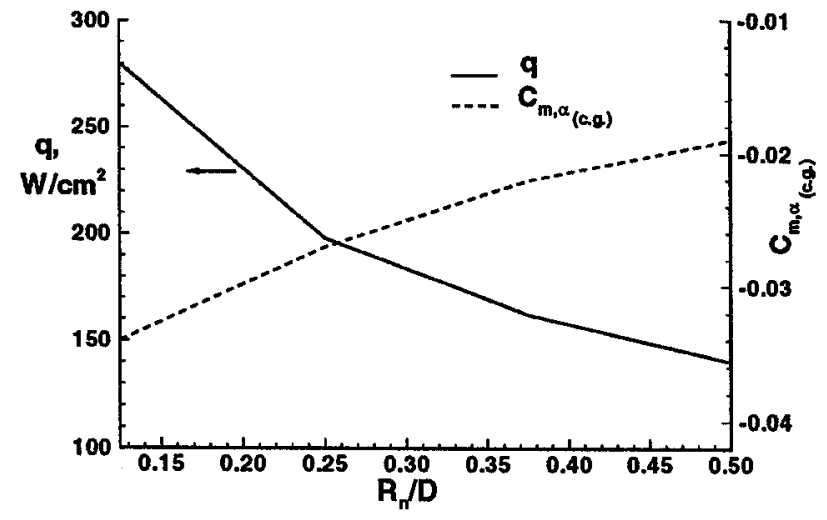

Fig. 2 Increased nose radius trade between maximum stagnation-point heating rate and transonic static stability.

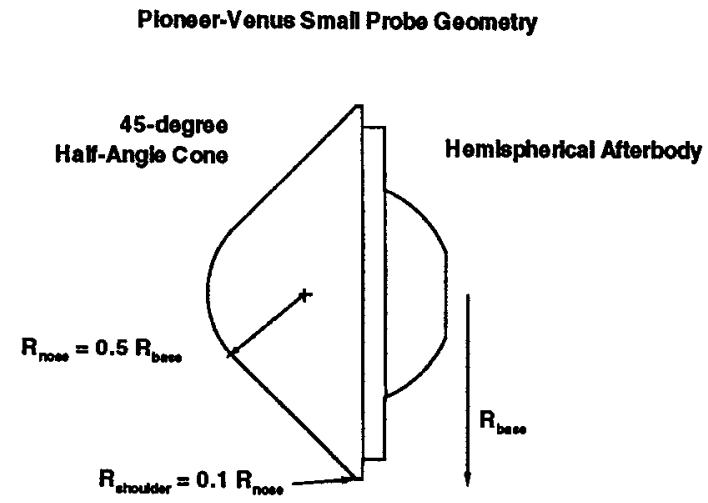

Fig. 3 Pioneer-Venus small probe geometry.

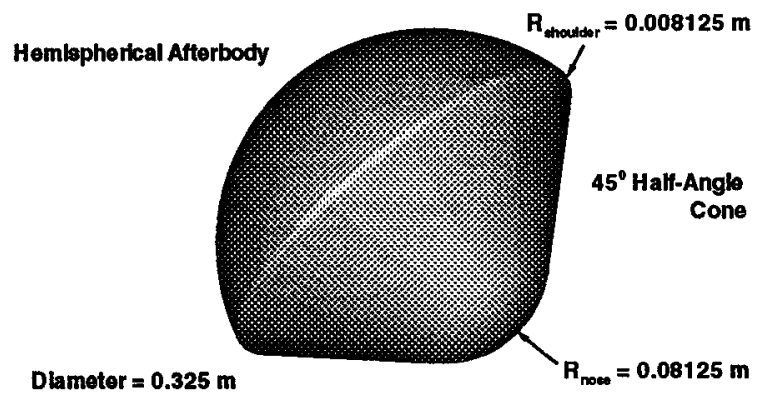

Fig. 4 Mars Microprobe aeroshell geometry.

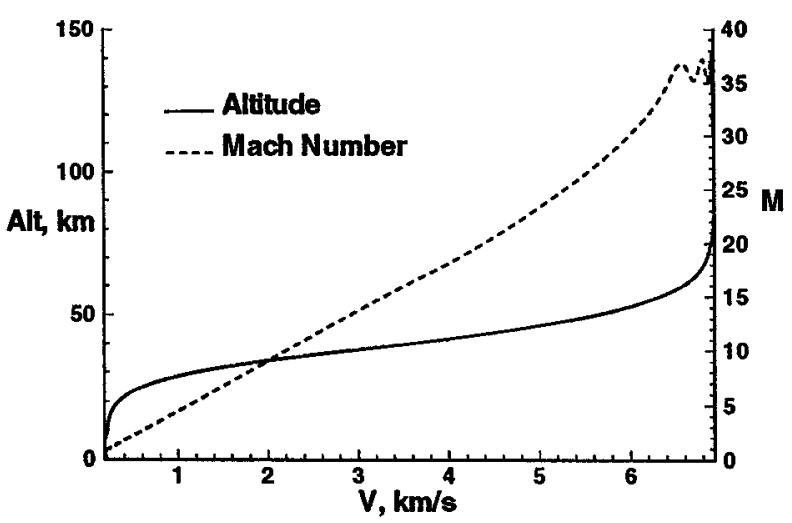

Fig. 5 Estimated Mars-entry trajectory.

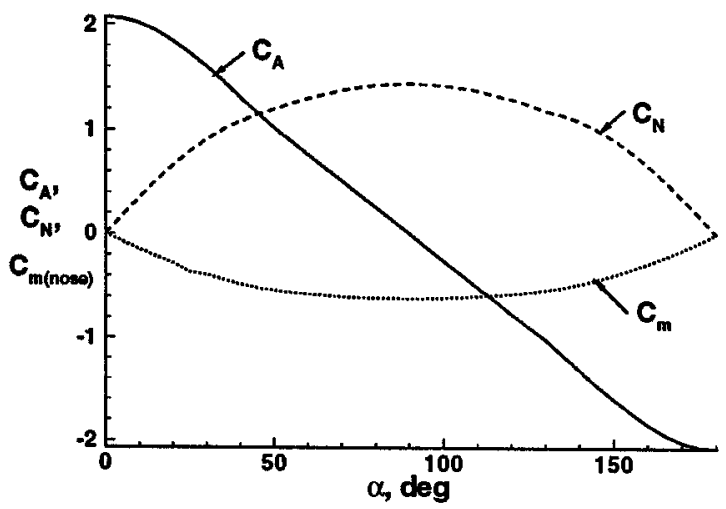

Fig. 6 Free-molecular flow aerodynamics. 


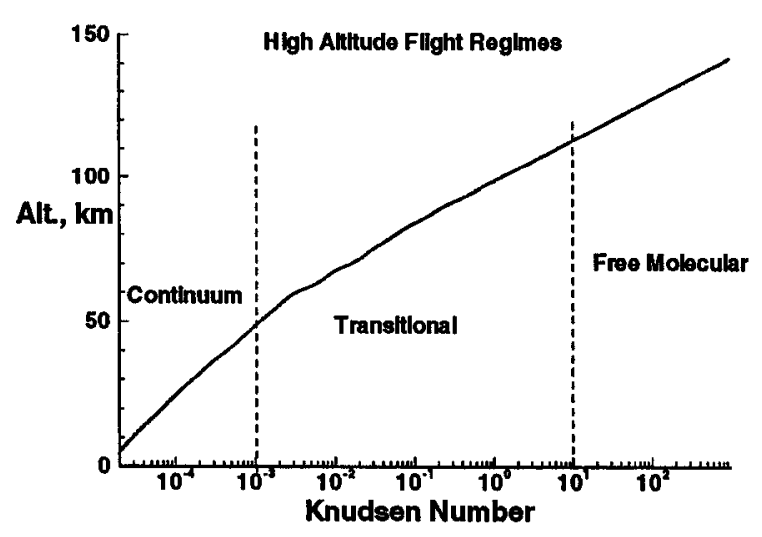

Fig. 7 High altitude flight regimes.

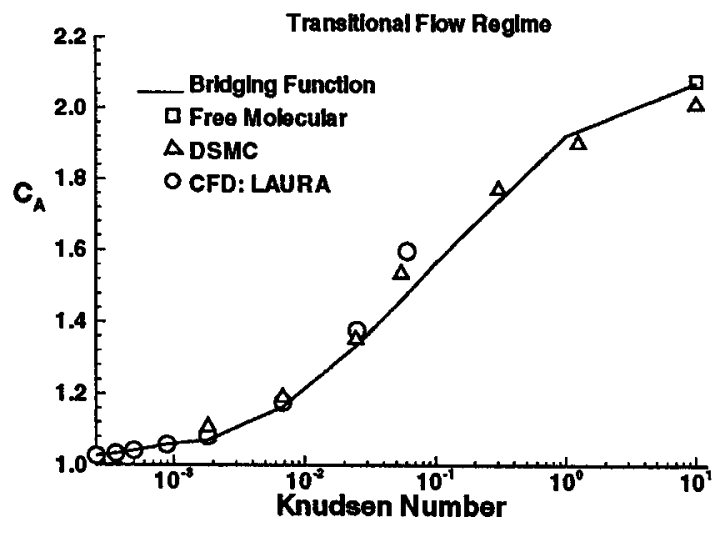

Fig. 8 Comparison of zero angle-of-attack axialforce coefficients in the transitional-flow regime.

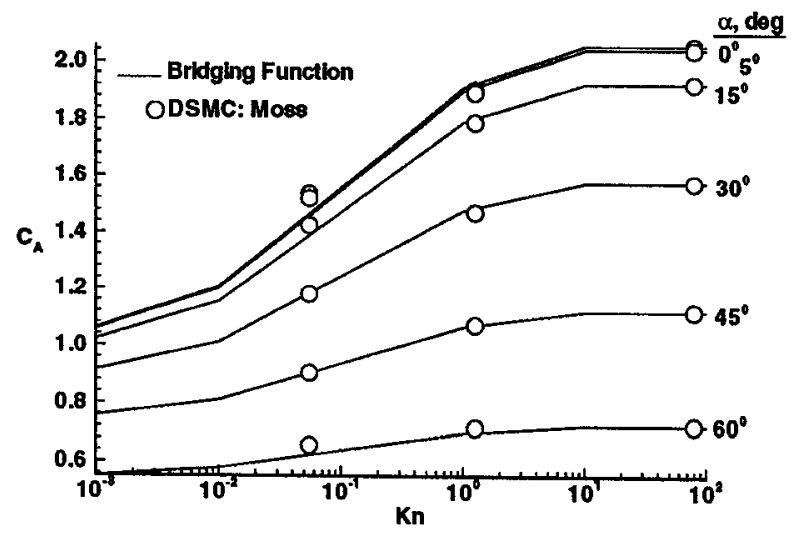

Fig. 9 Comparison of axial-force coefficients in the transitional-flow regime.

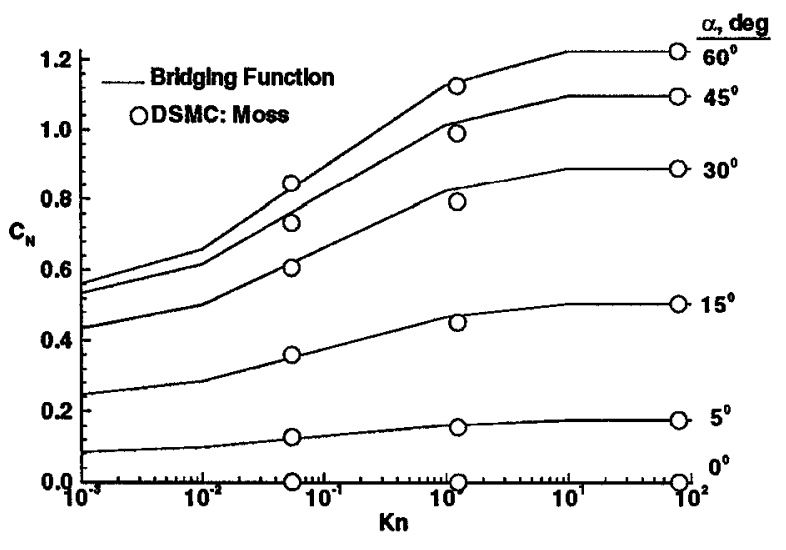

Fig. 10 Comparison of normal-force coefficients in the transitional-flow regime.

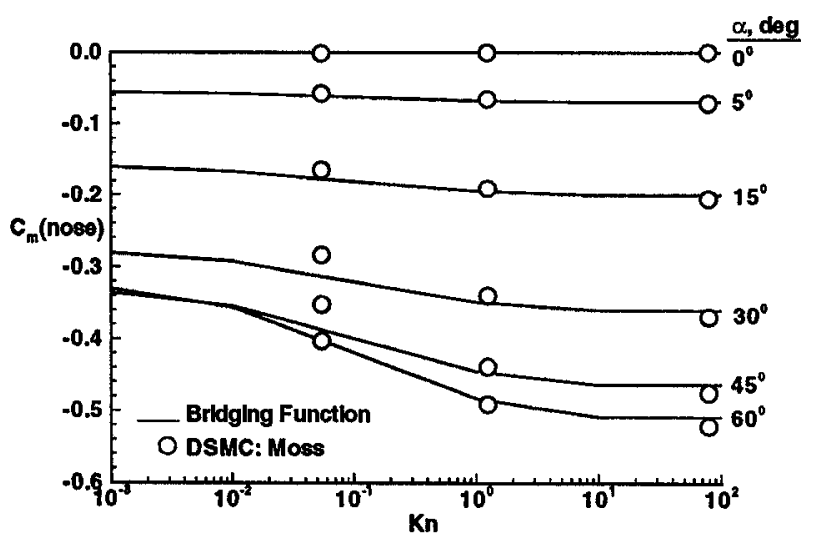

Fig. 11 Comparison of moment coefficients (about nose) in the transitional-flow regime.

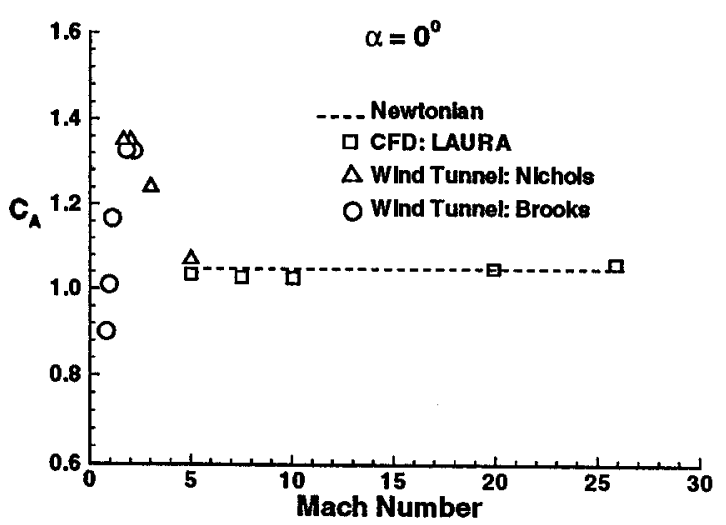

Fig. 12 Variation of axial-force coefficients in the hypersonic-flow regime. 


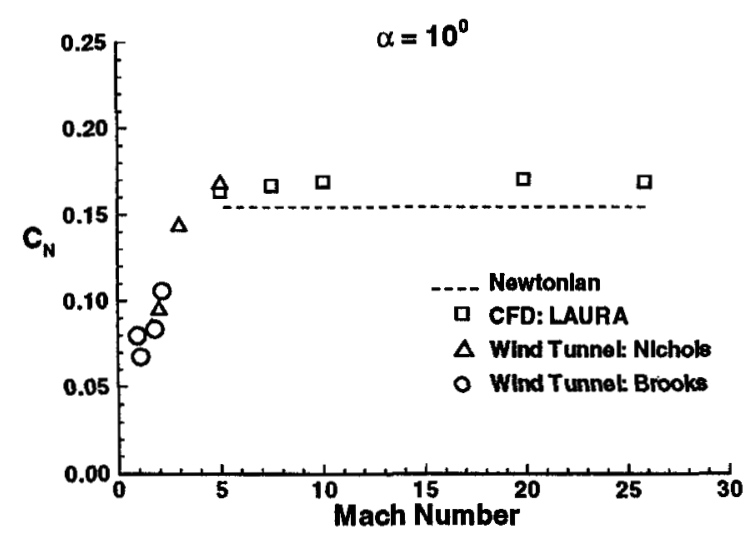

Fig. 13 Variation of normal-force coefficients in the hypersonic-flow regime.

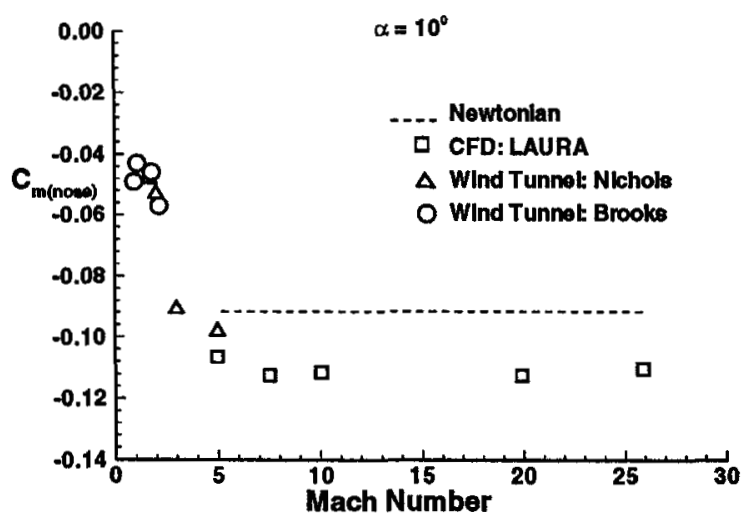

Fig. 14 Variation of the moment coefficient in the hypersonic-flow regime.

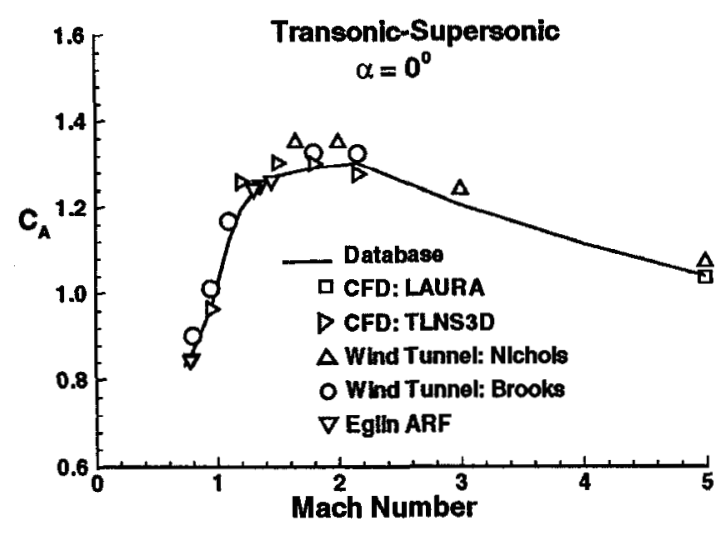

Fig. 16 Comparison of axial-force coefficient predictions in the transonic and subsonic regimes.

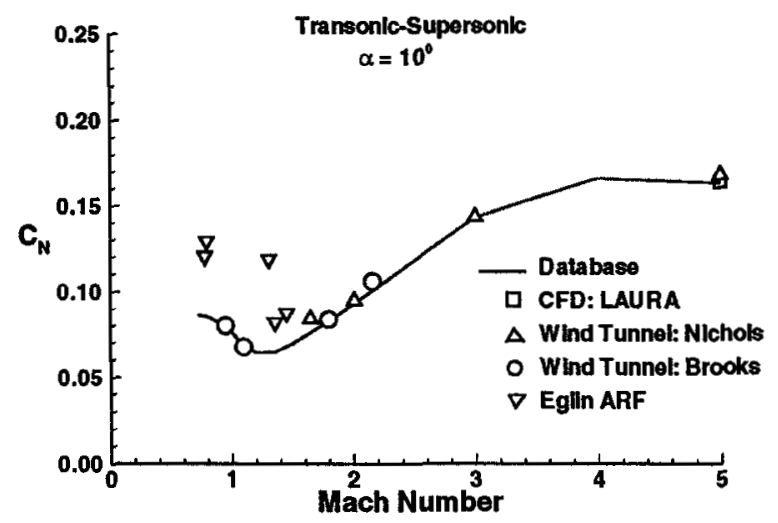

Fig. 17 Comparison of normal-force coefficient predictions in the transonic and subsonic regimes.

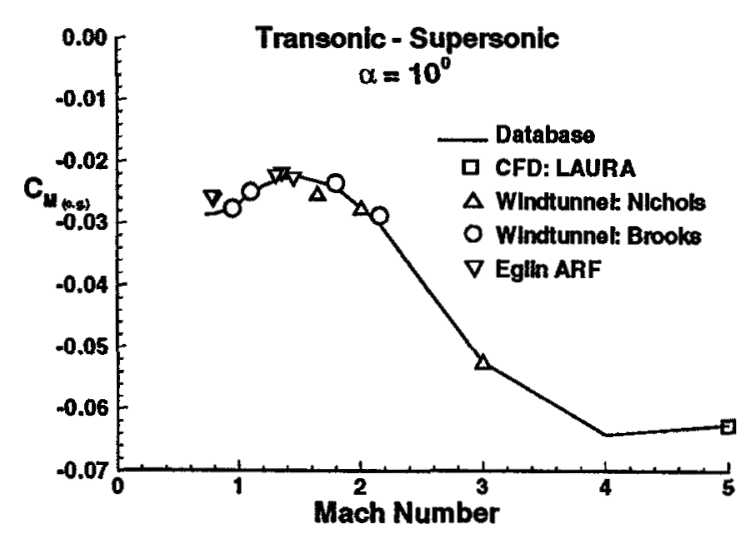

Fig. 18 Comparison of the moment coefficient predictions in the transonic and subsonic regimes. 


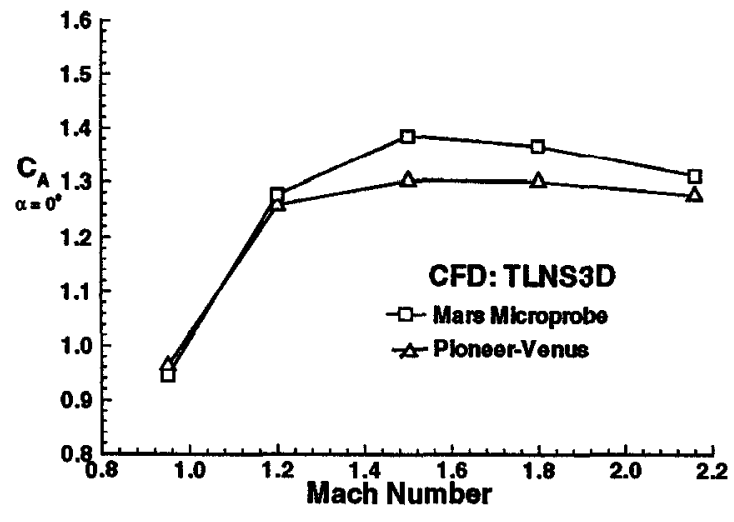

Fig. 19 Comparison of the TLNS3D CFD predictions for axial-force coefficient on different afterbodies.

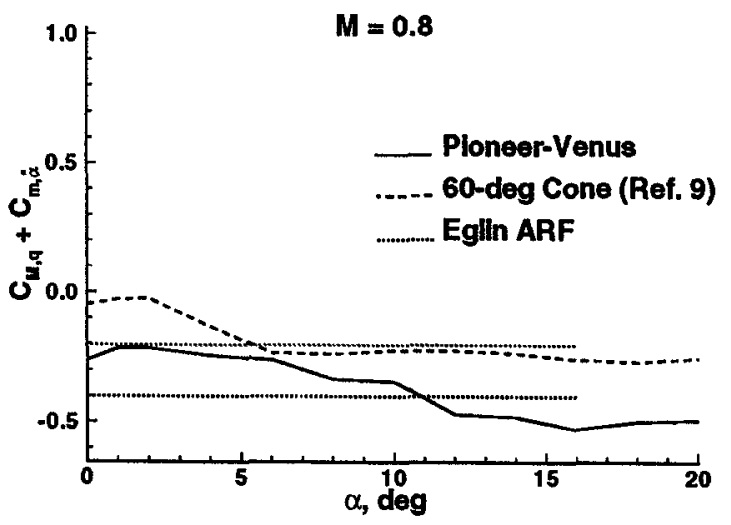

Fig. 20 Comparison of dynamic derivatives in the transonic regime $(M=0.8)$.

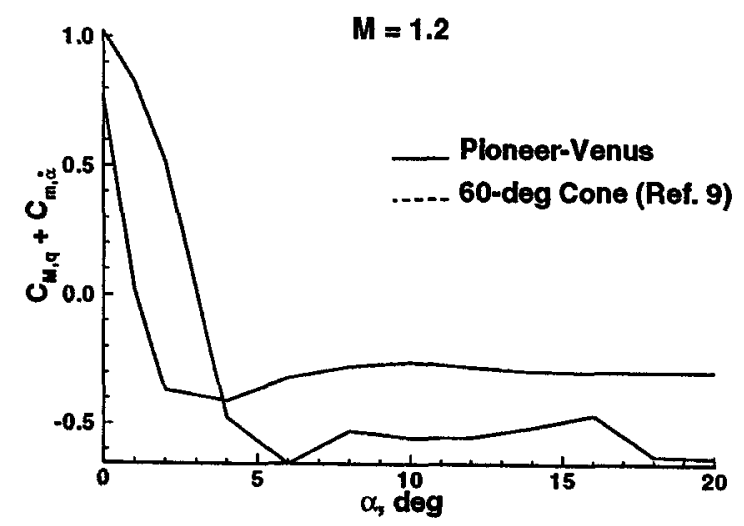

Fig. 21 Comparison of dynamic derivatives in the transonic regime $(M=1.2)$.

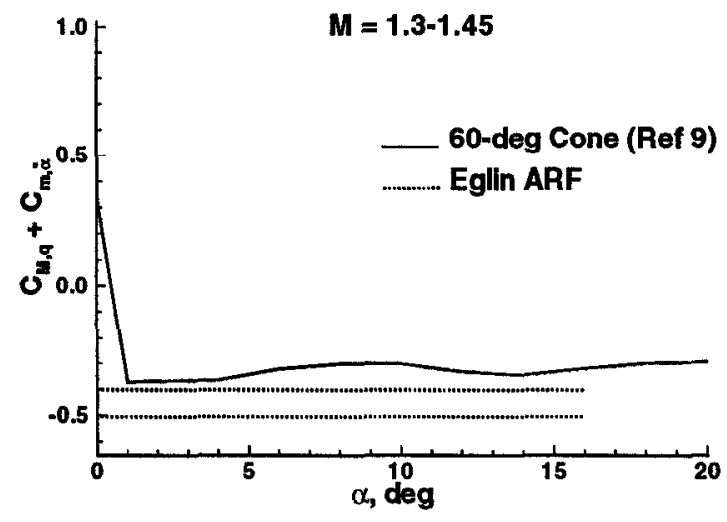

Fig. 22 Comparison of dynamic derivatives in the transonic regime $(M=1.3-1.45)$. 